\title{
Upper-Limb Exercises for Stroke Patients through the Direct Engagement of an Embodied Agent
}

\author{
Hee-Tae Jung, Jennifer Baird, Yu-Kyong Choe, and Roderic A. Grupen \\ University of Massachusetts Amherst \\ Amherst, MA 01003, USA \\ hjung@cs.umass.edu, bupt95@kin.umass.edu, ychoe@comdis.umass.edu, \\ grupen@cs.umass.edu
}

\begin{abstract}
In this case study, we examine the functional utility of an embodied agent as an interactive medium in stroke rehab. A set of physical rehab exercises is conducted through the direct engagement of an embodied agent, the uBot-5. Based on the preliminary data, we argue that a general-purpose embodied agent has a potential to functionally complement human therapists in providing rehab to stroke patients.
\end{abstract}

\section{Categories and Subject Descriptors}

J.3 [Computer Applications]: Life and Medical Scienceshealth; K.4.2 [Computers and Society]: Social Issuesassistive technologies for persons with disabilities

\section{General Terms}

Experimentation, Human Factors

\section{Keywords}

Autonomous Agents, Embodied Agent, Healthcare, Physical Rehab, Stroke, Telepresence

\section{INTRODUCTION}

Stroke is one of the leading causes of death in many countries. Those who survive from stroke experience devastating deficits such as hemiparesis. To alleviate impairments, patients pursue therapy services at the acute, sub-acute, and chronic phases as necessary. Patients need longitudinal rehab to sustain and improve motor functions even after they are discharged from hospitals or clinics. Stroke survivors living in federally designated health professional shortage areas need quality rehab services. Accordingly, it is critical to examine possible therapy methods that are applicable in residential settings.

In recent years, the potential of robot-mediated rehab has been widely acknowledged. Most studies employ specially designed robots with hardware components where patients can place their impaired limbs during the exercises [1]. These robots assist the movements of patients' impaired limbs while simulated targets are presented on a computer monitor screen. However, empirical study suggests that an embodied agent can induce more attention than can a simulated agent when engaging humans [2]. In addition, the com-

Copyright is held by the author/owner(s). HRI'11, March 6-9, 2011, Lausanne, Switzerland. ACM 978-1-4503-0561-7/11/03.

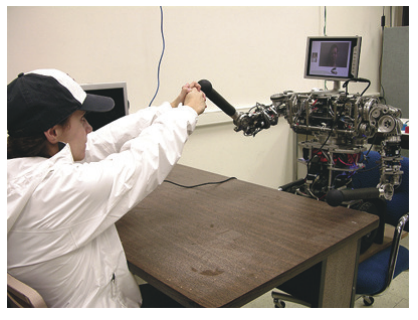

(a) Task 1

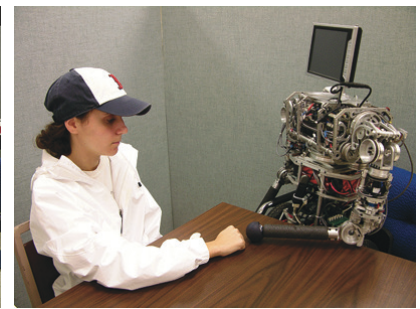

(b) Task 2
Figure 1: Research assistant demonstrating the exercise tasks.

parative studies suggest that patients can achieve significant improvements as long as they engage in well-planned, repetitive, and intensive exercises regardless of training medium [3]. Based on these findings, we seek a novel use of an embodied agent that can complement other contemporary approaches.

In the future, we envision that embodied agents will assist daily human activities and provide necessary healthcare services in residential settings [4]. Towards that end, it is important to examine if an embodied agent is functionally capable of providing therapeutic services. Specifically, in this case study, we study if an embodied agent can provide intensive upper-limb exercises to stroke patients.

\section{STUDY SETUP}

The current study design is approved by University of Massachusetts Amherst (UMass Amherst) Institutional Review Board.

\subsection{Human Subject}

The subject is a 56-year-old male who suffered from a stroke 15 years ago. He has right hemiparesis and ambulates with a quad cane. He has limited scapula and minimal shoulder and elbow active motion, with no use of the right hand. His right arm is essentially non-functional. Before starting the study, consent was obtained in written form. Then a therapist administered the Fugl-Meyer Assessment to assess his motor function. He scored 17 points in the assessment $^{1}$.

\footnotetext{
${ }^{1}$ The Fugl-Meyer Assessment consists of 32 items that evaluate upper extremity functions of stroke patients. A scale with scores for upper extremity impairment ranges from 0 (no function at all) to 66 (normal function).
} 


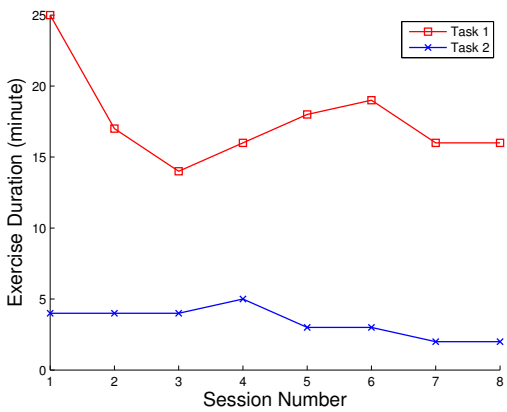

(a)

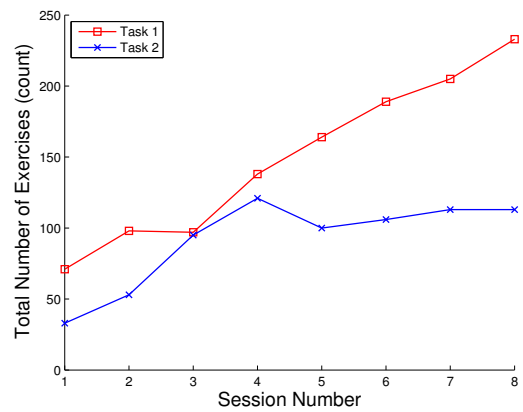

(b)

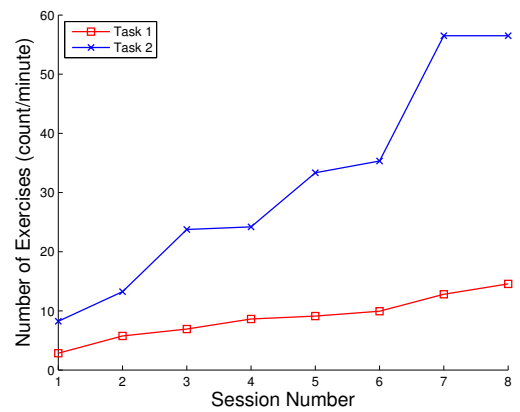

(c)

Figure 2: Experiment results show an observable increase in the number of trials across sessions.

\subsection{Robot Platform}

The uBot-5, a small light-weight bi-manual mobile manipulator developed at the Laboratory for Perceptual Robotics at UMass Amherst [5], delivered physical therapy. It was designed to be safe when assisting the elderly living in residential settings on daily basis, which was one of the reasons for being used in this study. To reduce the risks from unexpected accidents, throughout the therapy sessions, the robot sat across a table and thus a patient was always out of the robot's reach as shown in Fig. 1. Microsoft Robotics Developer Studio (MRDS) was used for the software environment.

\subsection{Rehab Therapy}

During the therapy design, we kept a human physical therapist in the loop to take advantage of the rich knowledge on traditional therapies proven to be effective and to apply this knowledge to robotic technology. Physical exercise tasks were determined based on the subject's current level of motor functions and through discussion between the clinician and the participant. The determined tasks were as follows.

1. Holding two hands together and stretching arms to reach for robot's hand presented at various points on the vertical plane; see Fig. 1a.

2. Without moving torso, reaching for robot's hand with patient's right hand placed on the table; see Fig. $1 \mathrm{~b}$.

For task 1, the uBot-5 was given a pool of desirable hand positions as task targets and programmed to choose a random sequence of the positions to promote a large enough range of motion. For task 2, the uBot-5 employed only a single target position. Due to the severe impairments of the subject, the main focus was placed on the range of motion. Exercise tasks were designed to be challenging but attainable with effort. A sequence of task 1 and task 2 was grouped into a single exercise set. Although 10 minutes for task 1 and 5 minutes for task 2 were assigned, the subject was given total control over the exercise duration for each task and the number of sets conducted.

\section{ANALYSIS}

The study consisted of 8 sessions in total. As can be seen in Fig. 2a, the time spent in the two exercise tasks did not increase across the sessions. In contrast, Fig. $2 \mathrm{~b}$ shows that the total number of trials completed for both tasks more than tripled. Thus, as can be seen in Fig. 2c, the subject demonstrated improvement in the average number of trials accomplished per minute. The average number of reaching movements increased 5 times for task 1 and more than 7 times for task 2. This preliminary data suggest that the efficiency of the subject's upper extremity movements can be enhanced through the direct engagement of an embodied agent. Further data are necessary to examine whether therapy-induced improvements can be observed in formal tests, e.g. Fugl-Meyer Assessment (FMA) or Wolf Motor Function Test (WMFT), and daily activities. It is interesting to note that the subject was willing to engage the therapy exercises even though social stimuli were not implemented in the uBot-5.

\section{ACKNOWLEDGMENTS}

This research was funded in part by a gift from Microsoft Corporation, ONR MURI award N00014-07-1-0749 and the Faculty Research Grant from UMass Amherst to Yu-Kyong Choe. Hee-Tae Jung acknowledges Robin Popplestone Fellowship and Jeong Song Culture Foundation Scholarship. The authors appreciate Megan Cronin for her help in conducting experiments and processing data.

\section{REFERENCES}

[1] L. Marchal-Crespo and D. J. Reinkensmeyer. Review of control strategies for robotic movement training after neurologic injury. International Journal of NeuroEngineering and Rehabilitation, 6(20), 2009.

[2] M. J. Matarić, et al. Socially assistive robotics for post-stroke rehabilitation. International Journal of NeuroEngineering and Rehabilitation, 4(5), 2007.

[3] A. C. Lo, et al. Robot-assisted therapy for long-term upper-limb impairment after stroke. The New England Journal of Medicine, 362(19):1772-1783, 2010.

[4] P. Deegan, et al. Mobile manipulators for assisted living in residential settings. Autonomous Robots, 24(2):179-192, 2007.

[5] P. Deegan, et al. Designing a self-stabilizing robot for dynamic mobile manipulation. In Workshop on Manipulation for Human Environments, Robotics: Science and Systems, Philadelphia, Pennsylvania, August 2006. 